\title{
Rapport sur la Science et la Technologie
}

Le Comité interministériel du 15 juillet 1998, à l'initiative du ministre de l'Éducation nationale, de la Recherche et de la Technologie, a confié à l'Académie des sciences l'établissement du rapport biennal sur l'état de la science et de la technologie.

Depuis 1999, trente rapports ont ainsi été édités et remis au ministre délégué à la Recherche.

Dans le cadre des liens étroits de collaboration entre l'Académie des sciences et l'Académie des technologies, il a été décidé de traiter le thème de la métallurgie. Un groupe de travail commun a été constitué. II a rédigé le présent rapport qui a été soumis aux procédures de validation propres à chacune des deux académies.

À l'Académie des sciences, le rapport a été soumis au Comité "Rapport Science et Technologie » (RST) le 19 janvier 2010, puis présenté à un Groupe de lecture critique le 9 avril 2010 et voté en séance plénière le 6 juillet 2010.

À l'Académie des technologies, le rapport a été présenté au Comité des travaux le 7 juin 2010, puis soumis au Comité de la qualité le 14 juin 2010 et voté par l'assemblée de l'Académie le 7 juillet 2010. 


\section{KIVSDJHIQMQWRQDQIOTKEOQN}

